\title{
Effects of Perfluorination on Thiophene and Pyrrole Oligomers
}

\author{
Ulrike Salzner $^{\dagger}$ \\ Department of Chemistry, Bilkent University, 06800 Bilkent, Ankara, Turkey \\ Received: January 20, 2010; Revised Manuscript Received: February 26, 2010
}

\begin{abstract}
The effect of perfluorination on thiophene and pyrrole oligomers in neutral, cationic, and anionic states was investigated with density functional theory at the (TD)B3P86-30\%/6-31G* level. For the title compounds fluorination leads to planarization. For pyrroles a band gap reduction of $0.58 \mathrm{eV}$ results, as unsubstituted pyrroles are nonplanar and disordered in the solid state. For thiophene the band gap is slightly increased as long thiophene oligomers are almost planar. Ionization energies and electron affinities increase upon fluorination by 0.65 and $0.60 \mathrm{eV}$ for polythiophene and by 0.45 and $0.90 \mathrm{eV}$ for polypyrrole. Conduction band widths increase by 0.5 for polythiophene and by $0.7 \mathrm{eV}$ for polypyrrole. Spectra of charged (doped) forms are almost identical to those of the parent systems. Like parent systems, fluorinated oligomers with chain lengths of more than six rings develop a third UV absorption that increases in strength and decreases in energy upon chain length increase.
\end{abstract}

\section{Introduction}

Almost since the beginning of research on conducting organic polymers, it was predicted that substitution with fluorine or electronegative groups would improve charging capacity and stability of n-doped forms. Theoretical studies on polyacetylene confirmed that planar perfluoropolyacetylene would have a high electron affinity and therefore the capacity to be an n-type conductor. ${ }^{1-3}$ However, the early theoretical research was based on nonoptimized geometries and ignored possible geometry changes due to steric and electrostatic interactions. Indeed, fluorination of acetylene oligomers ${ }^{4-6}$ leads to deviation from planarity although to a lesser extent than cyano-substitution., Theoretical comparison of the effects of cyano- and fluorosubstitution $^{4,5,7,8}$ revealed other interesting differences between $\mathrm{F}$ and $\mathrm{CN}$ as fluorine acts by an electron withdrawing inductive effect but is a $\pi$-donor, while the cyano group provides a low lying $\pi^{*}$-orbital and is therefore a $\pi$-acceptor in the first excited state or in n-doped systems. As a result, fluorine tends to increase conduction bandwidth, ${ }^{5}$ while cyano groups tend to produce low lying but localized energy levels within the band gap. ${ }^{9}$ Thus fluorinated polymers are more likely to have high n-type conductivities than cyano-substituted ones.

Attempts to synthesize perfluorinated polyacetylene put an end to speculations regarding possible improved properties, as only a highly air sensitive, ill-characterized material was obtained. ${ }^{10}$ In contrast, fluorination of polypyrrole succeeded, ${ }^{11}$ and the expected changes in electronic structure are borne out. Polydifluoropyrrole (PDFP) shows good conductivity $(\sim 0.1 \mathrm{~S}$ $\mathrm{cm}^{-1}$ ), a higher redox potential compared to that for polypyrrole (PP), and increased p-doping capacity (up to 55\%). Fluorination of other conjugated oligomers and polymers generated similar effects. Perfluorinated oligo( $p$-phenylenes $) \mathrm{s}^{12}$ and oligofluorenes ${ }^{13}$ are efficient n-type conductors; perfluorinated sexithiophene $^{14,15}$ (6DFT) has increased reduction and oxidation potentials compared to sexithiophene $(6 \mathrm{~T})^{14}$ and adopts a $\pi$-stacked rather than a herringbone crystal structure. ${ }^{15}$ The effect of perfluorination on the band gap varies as PDFP has a $0.6 \mathrm{eV}$ reduced band gap compared to that for $\mathrm{PP},{ }^{11}$ fluorinated

\footnotetext{
† E-mail: salzner@fen.bilkent.edu.tr.
}

polyaniline has a similar band gap as the parent polyaniline, ${ }^{16}$ 6DFT has slightly $(0.1-0.2 \mathrm{eV})$ increased absorption and emission maxima compared to those for $6 \mathrm{~T}$, and perfluorinated polyphenylene and polythienylene vinylenes exhibit a $0.9 \mathrm{eV}$ blue shift of absorption and emission maxima. ${ }^{17,18}$ Substitution and copolymerization of conducting polymers with fluorinated substituents or side chains likewise increases their electron affinities and therefore stabilizes n-doped forms. These systems are used for organic field effect transistors. ${ }^{19-28}$ Thus property changes upon fluoro-substituion are intriguing and of increasing importance for applications. A theoretical study on the intrinsic electronic properties of fluorinated thiophene and pyrrole oligomers and of their doping behavior was therefore carried out.

\section{Methods}

Thiophene and pyrrole oligomers with 1, 2, 6, 12, and 18 rings, with and without fluoro-substitution in all $\beta$-positions were optimized in neutral form, as cations, and as anions. Unsubstituted thiophene oligomers are abbreviated as $n \mathrm{~T}$, oligopyrroles as $n \mathrm{P}, n$ being the number of repeat units. Oligomers with difluorinated rings are abbreviated as $n \mathrm{DFT}$ and $n \mathrm{DFP}$. For polymers $n$ is replaced with P. Geometry optimizations were done with density functional theory employing the B3P86 hybrid functiona $\mathrm{l}^{29}$ with $30 \% \mathrm{HF}$ exchange ${ }^{30}$ (B3P86-30\%) and 6-31G* basis sets.

Comparison with our earlier studies on the parent systems, employing pseudopotentials, ${ }^{31,32}$ show very little difference between the two basis sets. LUMO levels tend to lie about 0.2 $\mathrm{eV}$ higher with $6-31 \mathrm{G}^{*}$, and the bond length alternation is slightly larger. Geometries agree slightly better with experiment and rotational barriers increase somewhat with $6-31 \mathrm{G}^{*},{ }^{33}$ which is the reason why this basis set was chosen for the present investigation. Because fluorine is involved, the effect of diffuse functions was tested with the $6-31+\mathrm{G}^{*}$ basis set on $2 \mathrm{DFP}$ and 6DFP. Diffuse functions lower the first excitation energy by $0.16 \mathrm{eV}$ for $2 \mathrm{DFP}$ and by $0.05 \mathrm{eV}$ for $6 \mathrm{DFP}$. The effect thus decreases for longer oligomers. For 2DFP four unoccupied $\sigma$-levels lie within the energy gap of the $\pi$-orbitals, which is most likely an artifact. The calculations were therefore done 
without diffuse functions. That diffuse functions are not crucial for anions because of the large size of the systems was shown for thiophene oligomer anions previously. ${ }^{34}$

For dimers 2T, 2P, 2DFT, and 2DFP, rotational profiles were calculated, fixing the dihedral angle between the rings and optimizing all other parameters. Employing density functional theory (DFT) for this purpose was discouraged for some time, but it was shown subsequently that disagreements between DFT and ab initio methods arose from the use of insufficient basis sets with the ab initio methods and not from failures of DFT..$^{35,36}$

Vertical ionization energies (IE)s were calculated explicitly with the $\triangle \mathrm{SCF}$ method as energy differences between total energies of neutral forms and cations at the geometry of the neutral species. Hybrid functionals produce very accurate $\triangle \mathrm{SCF}$ IEs. ${ }^{37}$ Trends in IEs were compared with DFT orbital energies. Orbitals energies with hybrid functionals predict low IEs but usually reproduce trends correctly. ${ }^{37}$

Excitation energies of neutral forms, of cations, and of some anions were investigated explicitly with excited state calculations. For neutral species time-dependent Hartree-Fock (TDHF/ 6-31G*) and TDB3P86-30\%/6-31G* levels were compared. TDHF and TDDFT methods ${ }^{38-41}$ are used as implemented in Gaussian03. ${ }^{42}$ With DFT spin contamination in ground states of open-shell cations and anions is very small. The values for the longest oligomers range from 0.762 to 0.765 for thiophene anions and cations and from 0.756 to 0.760 for pyrrole anions and cations with and without fluorine substitution. Spin contamination of excited states is not available in Gaussian03. A recent analysis has shown that excited state calculations of openshell systems are "fraught with danger" but that excitations involving the semioccupied orbital are "completely reasonable" ${ }^{43}$ Since the low lying excitations of doped conjugated oligomers all involve the semioccupied orbitals, the present results should be reliable.

TDDFT is problematic for neutral oligomers, as it is known to underestimate energy gaps and excitation energies of neutral conjugated $\pi$-systems. ${ }^{44-49}$ No such error was found for ions. ${ }^{31,50-53}$ TDHF is usually very accurate for excitation energies of neutral $\pi$-systems and shows a better chain length dependence but suffers from spin contamination for open-shell systems. ${ }^{31,52,54}$ Since all differences between parent and fluorinated thiophene and pyrrole oligomers are quantitatively identical with TDDFT and TDHF methods, only the TDB3P86-30\%/ 6-31G* are reported in the following also for neutral systems. Extrapolated band gaps are therefore underestimated but energy differences between fluorinated and parent systems are correct.

For excited states of cations and anions, the geometries were optimized and excitation energies were calculated without optimization on the ground state structures of the charged species. Thus the excitation energies model absorption spectra of doped oligomers. TD calculations produce excitation energies and oscillator strength and show which electronic transitions contribute with a given weight to each peak. UV spectra are plotted with Gabedit. ${ }^{55}$ Stick spectra are convoluted to simulate experimental spectra. Peak widths at half-maximum are $0.05 \mathrm{eV}$.

Extrapolation of oligomer data was done by plotting data against inverse chain length and extrapolating with second degree polynomial fits. In most cases deviation from linearity is small but not negligible. The plots are included in the Supporting Information. All calculations were carried out with Gaussian 03. ${ }^{42}$

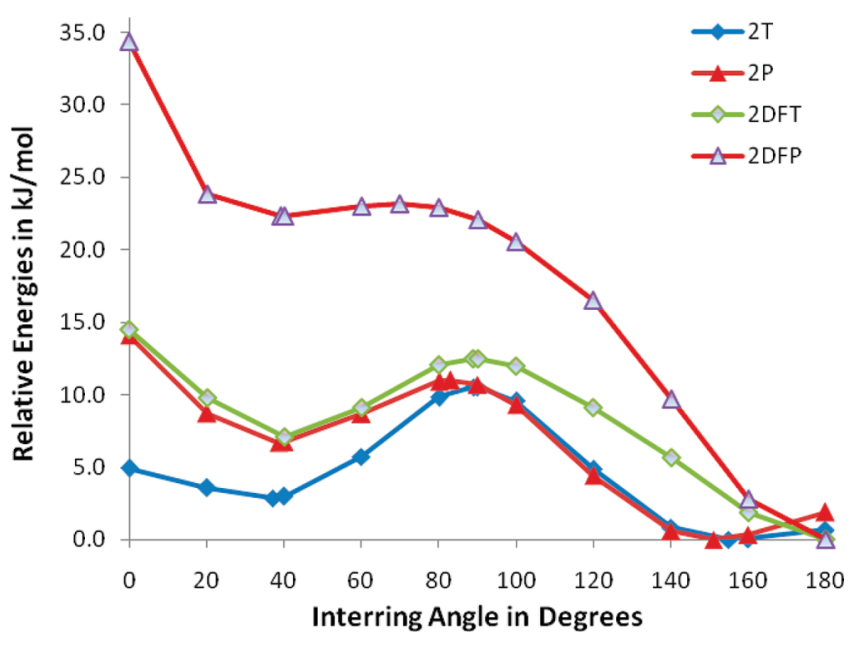

Figure 1. Rotational profiles for rotation around the inter-ring bond in $2 \mathrm{~T}, 2 \mathrm{DFT}, 2 \mathrm{P}$, and $2 \mathrm{DFP}$.

\section{Results}

Geometries. The first concern was to ensure that fluorine substitution at both $\beta$-positions does not induce distortion of the rings due to repulsion between the two F-atoms. Fluorinated thiophene and pyrrole monomers were verified to adopt planar structures by optimizing their geometries without symmetry constraints.

In Figure 1 rotational profiles are plotted for 2T, 2P, 2DFT, and 2DFP. There are two minima for each species. The lowest energy forms of unsubstituted $2 \mathrm{~T}$ and $2 \mathrm{P}$ are transoid minima with inter-ring angles of 155 and $151^{\circ}$, respectively. The cisoid minima have inter-ring angles of 37 and $39^{\circ}$ and lie higher in energy. The energy difference between transoid and cisoid structures is larger for $2 \mathrm{P}$ than for $2 \mathrm{~T}$. The shape of the rotational profile of the unsubstituted forms can be rationalized by steric repulsions that are expected to be larger for pyrrole than for thiophene oligomers since there is an additional $\mathrm{H}$-atom on the pyrrole nitrogen.

Fluorine substitution planarizes the dimers and the lowest energy forms have inter-ring dihedral angles of $180^{\circ}$. In addition, fluoro-substitution disfavors cisoid structures. In the case of 2DFP, the strong preference for the planar trans conformation can be attributed to hydrogen bonding between the $\mathrm{N}-\mathrm{H}$ hydrogen and the fluorine atom $\left(d_{\mathrm{F}-\mathrm{H}}=2.375 \AA\right)$. As a result, there is a steep energy increase for rotation around the interring bond. Planarization is also predicted for 2DFT; only the energy difference between trans and cisoid forms is much smaller compared to that for 2DFP in the absence of hydrogen bonding.

Upon chain length increase, twisting around the inter-ring bond vanishes for unsubstituted thiophene oligomers; only a slight bending (Figure 2) remains. The energy difference between planar and bent $18 \mathrm{~T}$ is $2.9 \mathrm{~kJ} / \mathrm{mol}$. Pyrrole oligomers are twisted at all chain lengths (Figure 2). The planarization energy for $18 \mathrm{P}$ is $19.6 \mathrm{~kJ} / \mathrm{mol}$.

Fluorinated oligomers have no tendency to twist around the inter-ring bonds, as indicated by the planar structures of dimers. There is also no bending in fluorinated thiophene species. Planarity of fluorinated oligomers was verified for 12DFT and 12DFP by geometry optimizations starting from twisted and bent forms, respectively, which resulted in fully planar optimized structures. Optimized structures of 18DFP and 18DFT are shown in Figure 3.

Ionization Energies. $\triangle$ SCF IEs and negative HOMO orbital energies of parent, fully optimized nonplanar forms and of planar 

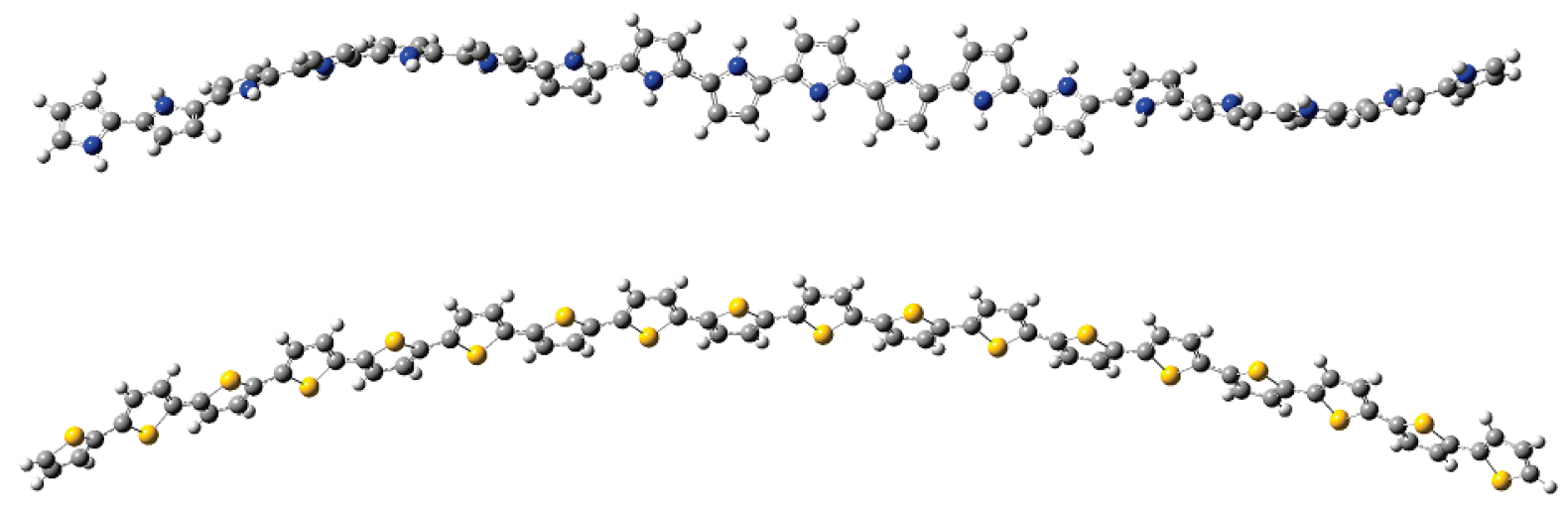

Figure 2. Fully optimized structures of $18 \mathrm{~T}$ (bottom) and 18P (top).
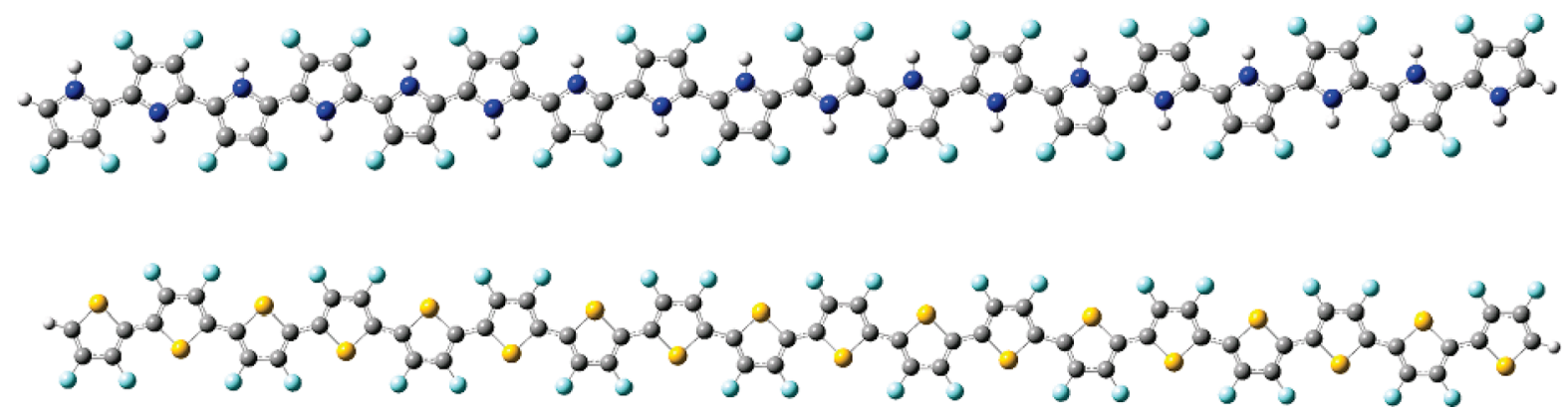

Figure 3. Fully optimized structures of 18DFT (bottom) and 18DFP (top).

TABLE 1: Ionization Energies and Negative HOMO Energies of Parent and of Fluorinated Thiophene and Pyrrole Oligomers

\begin{tabular}{lcccc}
\hline & \multicolumn{4}{c}{$\Delta$ SCF } \\
\cline { 2 - 5 } & $\mathrm{nT}$ & $\mathrm{nDFT}$ & $\mathrm{nP}$ & $\mathrm{nDFP}$ \\
\hline 2 & 8.10 & 8.58 & 7.38 & 7.87 \\
6 & 6.75 & 7.30 & 6.09 & 6.55 \\
12 & 6.33 & 6.90 & 5.67 & 6.13 \\
18 & 6.11 & 6.74 & 5.50 & 5.96 \\
$\infty$ & 5.79 & 6.44 & 5.19 & 5.64 \\
\hline \multicolumn{5}{r}{$-\varepsilon_{\text {HOMO }}$} \\
& \multicolumn{5}{c}{$\mathrm{n}$} \\
\hline 2 & 6.63 & 7.11 & 5.82 & 6.31 \\
6 & 5.88 & 6.43 & 5.14 & 5.62 \\
12 & 5.73 & 6.30 & 5.01 & 5.49 \\
18 & 5.71 & 6.27 & 5.00 & 5.46 \\
$\infty$ & 5.62 & 6.20 & 4.94 & 5.38
\end{tabular}

fluorinated oligomers are summarized in Table 1 and plotted in Figure 4 for thiophenes and in Figure 5 for pyrroles. Plots showing the extrapolation to infinite chain length are given as Supporting Information. Fluorination increases IEs by around $0.5 \mathrm{eV}$. For thiophene oligomers, the effect increases with chain length extension (2DFT $0.48 \mathrm{eV}$ and PDFT $0.65 \mathrm{eV}$ by extrapolating to infinite chain length). For pyrrole short and long oligomers show similar responses to fluorination (2DFP 0.49 and PDFP $0.45 \mathrm{eV}$ ). As usual, DFT underestimates IPs when orbital energies are used but the change upon fluorination is very similar to that with the $\triangle \mathrm{SCF}$ method. It is interesting to note that negative HOMO energies converge faster than $\triangle \mathrm{SCF}$ IPs so that both converge to very similar values in the long chain limit.

Electron Affinities. $\triangle$ SCF EAs and negative LUMO orbital energies of parent, fully optimized nonplanar forms and of planar fluorinated oligomers are summarized in Table 2. Plots showing the extrapolation to infinite chain length are given as Supporting

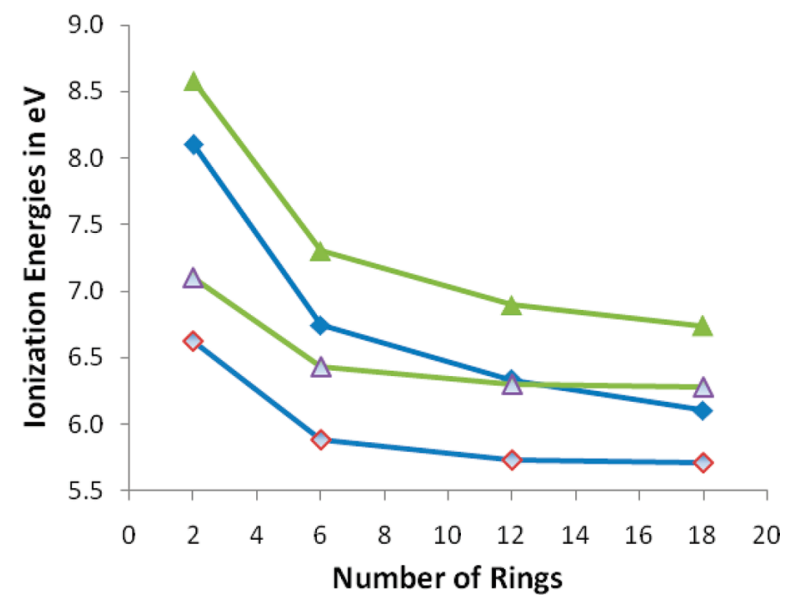

Figure 4. Ionization energies of thiophene oligomers (blue diamonds) and fluorinated thiophene oligomers (green triangles) calculated with the $\triangle \mathrm{SCF}$ method (filled symbols) and obtained as negative HOMO energies (open symbols).

Information. $\triangle \mathrm{SCF}$ EAs are slightly $(\sim 0.3 \mathrm{eV})$ lower than negative LUMO energies but the effects of fluorination are almost identical with both approaches. In the polymer limit PDFT is predicted to have a $0.66(0.67) \mathrm{eV}$ higher EA than PT and PDFP to have a 1.07 (1.14) eV higher EA than PP using the $\triangle \mathrm{SCF}$ method and orbital energies (in parentheses), respectively. The effects of fluorination on IP and EA are thus similar for PDFT but the effect is larger on the EA for PDFP.

Excitation Energies, Energy Gaps, and Bandwidth of Neutral Species. As fluorination leads to planarization of thiophene and pyrrole oligomers, excitation energies of unsubstituted oligomers were investigated for fully optimized nonplanar and for planar structures to separate geometric and electronic effects of fluorination. All excitation energies, including those of charged species are summarized in Table 3.

Figure 6 shows the trends in excitation energies of upon chain lengths increase for neutral thiophene oligomers. Because there 


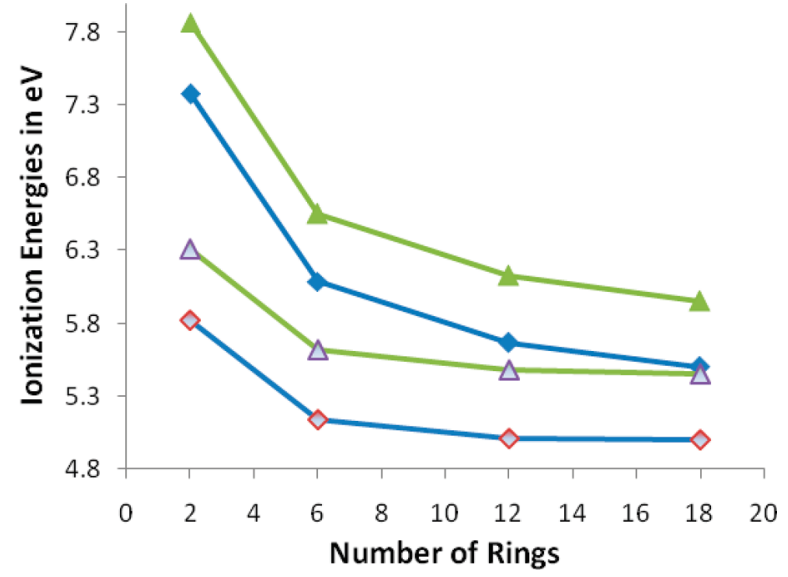

Figure 5. Ionization energies of pyrrole oligomers (blue diamonds) and fluorinated pyrrole oligomers (green triangles) calculated with the $\triangle \mathrm{SCF}$ method (filled symbols) and obtained as negative HOMO energies (open symbols).

TABLE 2: Electron Affinities and Negative LUMO Energies of Parent and of Fluorinated Thiophene and Pyrrole Oligomers

\begin{tabular}{lcrrr}
\hline & \multicolumn{5}{c}{$\Delta$ SCF } \\
\cline { 2 - 5 } & $\mathrm{nT}$ & $\mathrm{nDFT}$ & $\mathrm{nP}$ & $\mathrm{nDFP}$ \\
\hline 2 & 0.02 & 0.54 & -1.65 & -0.91 \\
6 & 1.60 & 2.21 & -0.18 & 0.79 \\
12 & 2.12 & 2.75 & 0.30 & 1.35 \\
18 & 2.30 & 2.96 & 0.49 & 1.56 \\
$\infty$ & 2.69 & 3.36 & 0.85 & 1.97 \\
\hline & \multicolumn{5}{c}{$-\varepsilon_{\text {LUMO }}$} \\
& $\mathrm{n}$ & $\mathrm{nDFT}$ & $\mathrm{nP}$ & $\mathrm{nDFP}$ \\
\hline 2 & 1.47 & 2.01 & -0.12 & 0.65 \\
6 & 2.47 & 3.08 & 0.78 & 1.73 \\
12 & 2.74 & 3.36 & 0.97 & 2.01 \\
18 & 2.79 & 3.44 & 1.01 & 2.08 \\
$\infty$ & 2.96 & 3.63 & 1.12 & 2.26
\end{tabular}

is no twisting between rings in the fully optimized structures of thiophene oligomers, planarized species have only slightly smaller excitation energies than fully optimized (bent) ones. Fluorination slightly increases excitation energies compared to those of planar oligomers. The excitation energies extrapolate to $1.95 \mathrm{eV}$ for parent PT, $1.83 \mathrm{eV}$ for planar PT, and to 1.90

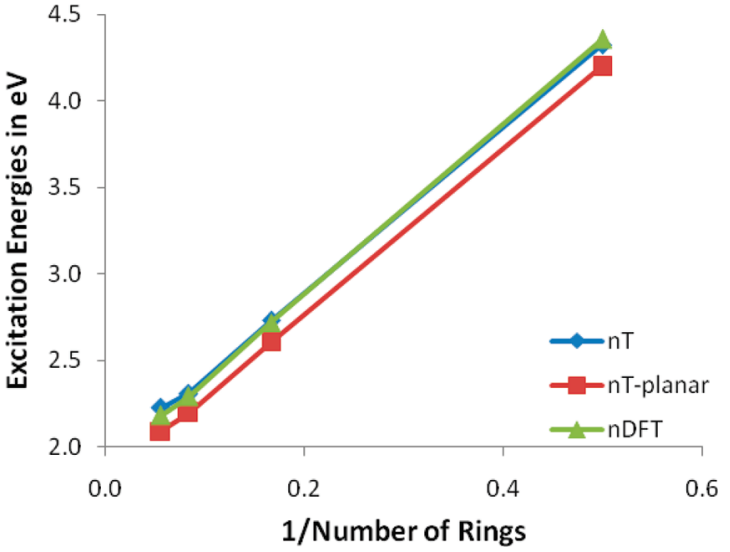

Figure 6. TDB3P86-30\%/6-31G* excitation energies for thiophene oligomers.

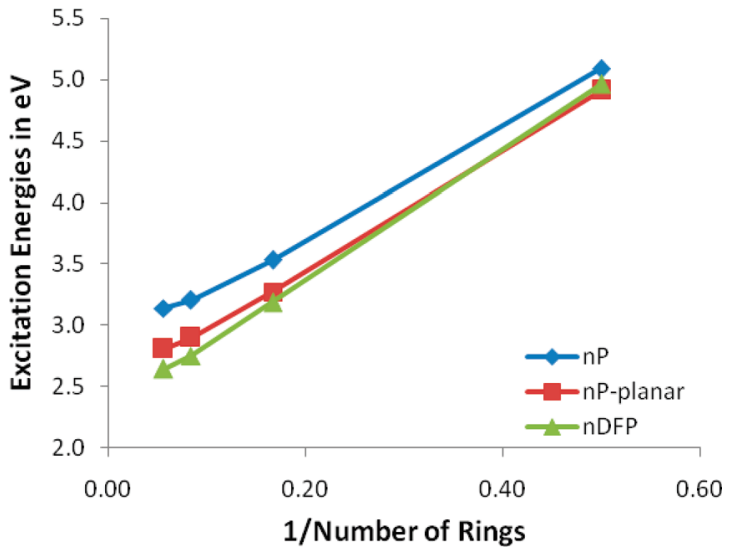

Figure 7. TDB3P86-30\%/6-31G* excitation energies for pyrrole oligomers.

for PDFT. DFT HOMO-LUMO gaps extrapolate to: 2.66, 2.52, and $2.57 \mathrm{eV}$. Fluorination thus lowers the band gap by about 0.05-0.10 eV compared to that for nonplanar PT and increases it by about $0.05-0.07 \mathrm{eV}$ compared to that for planar PT.

The effect of fluoro-substitution on neutral pyrrole oligomers is larger than that on thiophene oligomers. Figure 7 indicates that this is due to several factors. As parent pyrrole oligomers are twisted and fluorinated pyrroles are planar, there is a larger geometry effect on the band gap than for thiophene oligomers. Twisted oligomers converge faster to constant excitation energy

TABLE 3: Excitation Energies in eV, Oscillator Strengths (in Parentheses) of Thiophene and Pyrrole Oligomers

\begin{tabular}{|c|c|c|c|c|c|c|c|c|}
\hline & \multicolumn{2}{|c|}{ neutral } & \multicolumn{3}{|c|}{ cation } & \multicolumn{3}{|c|}{ anion } \\
\hline & opt & planar & E1 & E2 & E3 & E1 & $\mathrm{E} 2$ & E3 \\
\hline $2 \mathrm{~T}$ & $4.32(0.42)$ & $4.20(0.44)$ & $1.96(0.004)$ & $2.33(0.05)$ & $3.39(0.49)$ & $2.10(0.05)$ & $3.10(0.39)$ & \\
\hline $12 \mathrm{~T}$ & $2.31(4.34)$ & $2.20(4.47)$ & $0.47(1.76)$ & $1.37(1.38)$ & $2.22(1.33)$ & $0.47(1.63)$ & $1.38(1.49)$ & $2.25(1.07)$ \\
\hline $18 \mathrm{~T}$ & $2.23(6.54)$ & $2.09(6.92)$ & $0.27(2.01)$ & $1.25(0.61)$ & $2.04(4.58)$ & $0.27(1.93)$ & $1.26(0.77)$ & $2.04(4.30)$ \\
\hline 2DFT & & $4.36(0.47)$ & $1.66(0.02)$ & $2.31(0.06)$ & $3.50(0.49)$ & $2.20(0.06)$ & $3.27(0.47)$ & \\
\hline $6 \mathrm{DFT}$ & & $2.72(2.23)$ & $1.01(0.65)$ & $1.91(1.76)$ & & $1.00(0.61)$ & $1.90(1.77)$ & \\
\hline $2 \mathrm{P}$ & $5.09(0.57)$ & $4.91(0.61)$ & $2.43(0.03)$ & $2.57(0.09)$ & $4.04(0.55)$ & $1.94(0.07)$ & $3.36(0.27)$ & \\
\hline $6 \mathrm{P}$ & $3.53(1.98)$ & $3.27(2.11)$ & $1.02(0.80)$ & $2.48(1.26)$ & & $0.86(0.61)$ & $2.56(1.13)$ & \\
\hline $12 \mathrm{P}$ & $3.20(4.20)$ & $2.90(4.47)$ & $0.44(1.43)$ & $2.16(0.75)$ & $2.91(2.32)$ & $0.40(1.33)$ & $2.21(0.61)$ & $2.94(2.19)$ \\
\hline $18 \mathrm{P}$ & $3.14(6.46)$ & $2.81(6.92)$ & $0.26(1.47)$ & $2.08(0.38)$ & $2.76(5.31)$ & $0.23(1.17)$ & $2.11(0.36)$ & $2.78(5.22)$ \\
\hline 2DFP & & $4.96(0.65)$ & $2.06(0.04)$ & $2.61(0.07)$ & $4.08(0.59)$ & $2.30(0.08)$ & $3.81(0.32)$ & \\
\hline $6 \mathrm{DFP}$ & & $3.19(2.29)$ & $1.08(0.80)$ & $2.40(1.47)$ & & $1.00(0.68)$ & $2.49(1.39)$ & \\
\hline
\end{tabular}




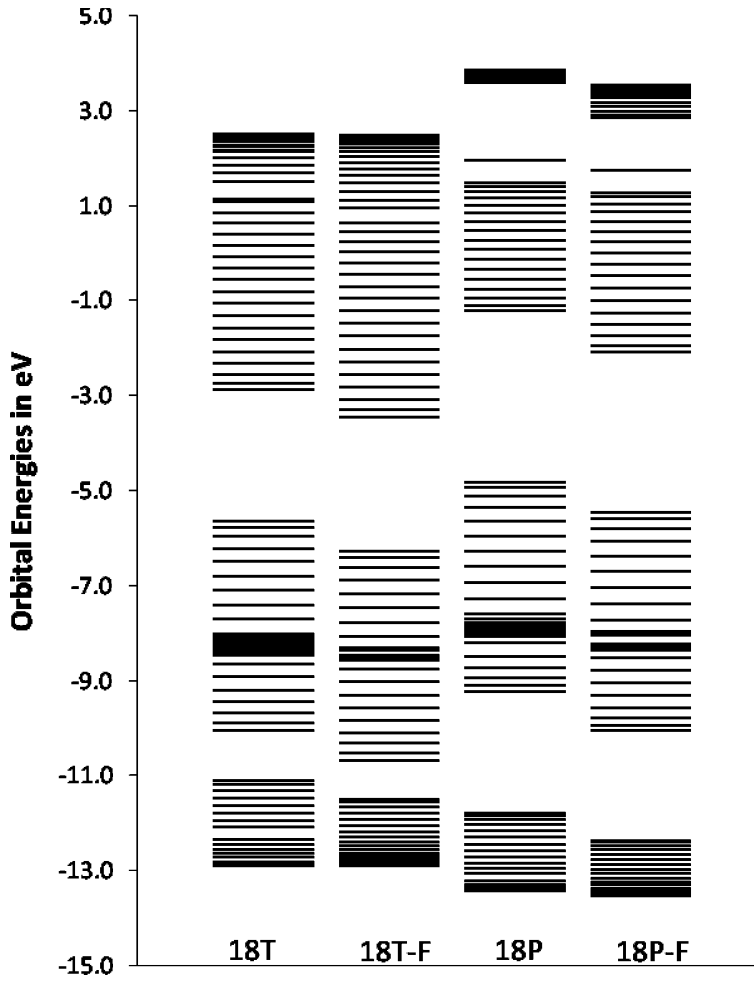

Figure 8. $\pi$-energy levels of 18 -mers of thiophene and pyrrole oligomers.

than planar ones with increasing chain length. Planarization without fluorination is predicted to decrease the band gap of PP by almost $0.4 \mathrm{eV}$. Fluorination slightly increases the excitation energy of $2 \mathrm{P}$ but excitation energies of fluorinated oligomers decrease faster and converge later upon chain length extension than those of nonfluorinated ones. Therefore, fluorination leads to an additional band gap decrease compared to that of planar PP. Overall, there is a predicted reduction of the band gap of PP of $0.58 \mathrm{eV}$ upon fluoro-substitution.

Band Widths. The larger decrease of excitation energies with increasing chain length of fluoro-substituted oligomers is due to stronger conjugation and is also reflected in increased conduction band widths. Band widths of bands originating from the five $\pi$-orbitals of thiophene and pyrrole monomers are shown in Figure 8 for 18T, 18P, 18DFT, and 18DFP. Pyrrole and thiophene oligomers have different band structures as there is a much larger gap between the lowest and highest $\pi$-bands in valence and conduction bands for pyrroles than for thiophenes. Thus three separate bands are formed for PT and four for PP. For pyrrole oligomers, where the conduction band is split into two parts, taking the lower part only, the bandwidth is considerably smaller than for thiophene oligomers.

Fluoro-substitution, nonetheless, influences both systems in the same way. The valence bands are pulled down in energy by $0.6 \mathrm{eV}$. Extrapolated to infinite chain length, the bandwidth of the valence band is unchanged for PT and increased by 0.2 $\mathrm{eV}$ for PP. The conduction bands are stretched upon fluorination as the low lying orbitals being influenced stronger than the high lying ones. For PT the conduction band edge is decreased by $0.6 \mathrm{eV}$ and the bandwidth is increased by $0.5 \mathrm{eV}$. For PP the decrease of the lower edge is $0.9 \mathrm{eV}$, and the increase of the band widths is $0.7 \mathrm{eV}$.

Excitation Energies of Charged Species. Excitation energies of cations and of anions are included in Table 3. In Figures 9 and $10 \mathrm{UV}$-absorption spectra of neutral fluorinated thiophene and pyrrole oligomers are plotted together with those of their cations and anions. UV-absorption spectra of fluorinated oligothiophene and oligopyrrole cations and anions are very similar to those of their unfluorinated parents. ${ }^{31,32,34,53}$

Cation dimers show three subgap peaks, the first two arising from mixing of $2-1$ and $4-1$ transitions of $\beta$-electrons. The third peak dominates the spectra and corresponds to a $1-1^{\prime}$ transition of an $\alpha$-electron. The numbers are obtained by labeling the highest occupied molecular orbital (HOMO) as " 1 " and the lowest unoccupied molecular orbital (LUMO) as " 1 "”. The following orbitals are labeled with increasing numbers. Thus $1-1^{\prime}$ is the HOMO-LUMO transition. For details regarding this nomenclature see refs 53 and 56. There is no analogue for the $4-1$ transition in anions. Two transitions predicted for anion spectra arising from $\alpha 1^{\prime}-2^{\prime}$ and $\beta 1-1^{\prime}$ transitions. The $2-1$ $\left(1^{\prime}-2^{\prime}\right)$ transitions of cations and anions have very low oscillator strength for dimers.

Upon chain length increase, the 4-1 transitions disappear and cation and anion spectra look very similar. Thus electronhole symmetry is preserved upon fluorination. The two peaks shift to lower energy and gain oscillator strength, so that medium-sized oligomers show the two typical subgap peaks observed upon doping of conducting polymers.

When chain lengths increase beyond 6-rings, configuration interaction sets in between the $1-1^{\prime}$ and $2-2,3-1^{\prime}$, and $1-3^{\prime}$ transitions. The reason for this is that energy levels get closer with increasing chain lengths and levels that are well separated for short oligomers lie very close for long ones. As a result, the $1-1^{\prime}$ transition mix with transitions that have high energies for short oligomers but relatively low energies for long ones. As a result, the peak splits into two major components E2 and E3 and depending on chain lengths some minor ones. With increasing chain length, the E2 peak decreases in intensity as the new peak E3 gains oscillator strength at its expense. For 12-mers E3 occurs almost at the same energy as the $1-1^{\prime}$ transition of the neutral species. The transition shifts slightly below the band gap of the neutral form for longer chains. It seems that the change from two to three sub-band peaks has been observed experimentally ${ }^{57}$ but was rationalized differently because its appearance is not expected according to the polaron model. The splitting of the $1-1^{\prime}$ into two components is due to configuration interaction (E2 and E3 are not dominated by a single electron transition) and therefore beyond the one-electron approximation. It is noteworthy that the described spectra do not change significantly if the ions are not optimized and the spectra are calculated at the geometries of the neutral species. Therefore, the appearance of subgap peaks upon doping is not related to geometric distortion as predicted by the polaron model $^{58}$ but is an electronic effect. The behavior is completely analogous to that of the parent systems. ${ }^{31,32}$

Compared to situations with unsubstituted oligomers, only minor changes in oscillator strengths and slight shifts in peak energies are predicted upon fluorination. The similarity between spectra of unsubstituted and fluorinated species is shown for hexamers in Figure 11. The low energy peaks are hardly affected and the high energy peaks are shifted up by $0.1 \mathrm{eV}$ for 6DFT and down by the same amount for 6DFP. Data in Table 3 show that the same is true for dimers and 12-mers. There is no strong geometry effect even for pyrrole cations since all cations (with and without fluorine substitution) are planar.

The low energy $1-2\left(1^{\prime}-2^{\prime}\right)$ transitions occur at almost the same energy for thiophenes and pyrroles no matter whether positively or negatively charged and do not change upon fluorosubstitution, as these transitions are related to the shift of the empty $\beta$-orbital relative to the valence (conduction) band upon 

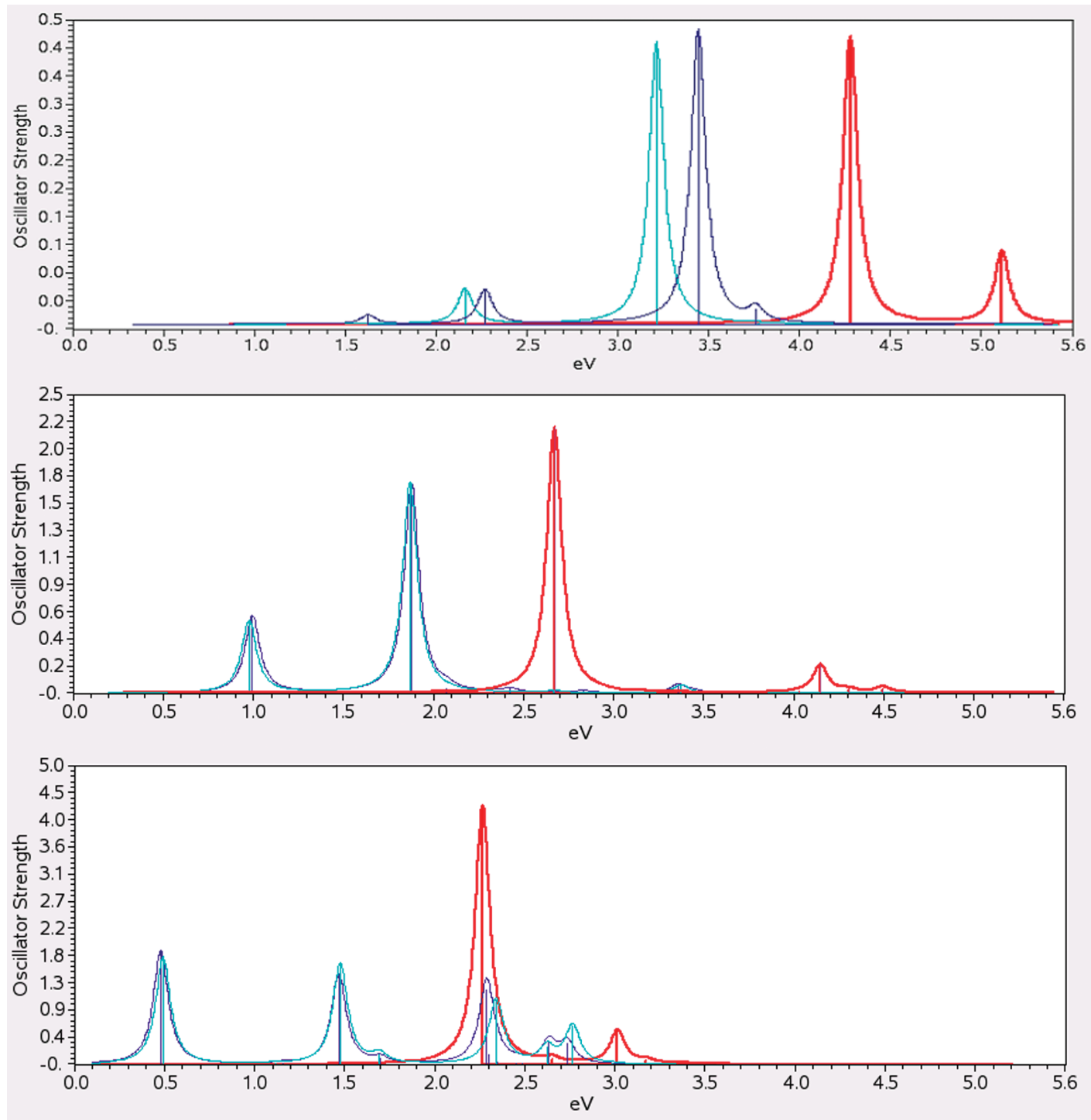

Figure 9. UV-absorption spectra of (top) $2 \mathrm{DFT}, 2 \mathrm{DFT}^{+}$, and $2 \mathrm{DFT}^{-}$, (middle) $6 \mathrm{DFT}, 6 \mathrm{DFT}^{+}$, and $6 \mathrm{DFT}^{-}$, and (bottom) $12 \mathrm{DFT}, 12 \mathrm{DFT}{ }^{+}$, and $12 \mathrm{DFT}^{-}$at the TDB3P86-30\%/6-31G* level of theory. All neutral species are shown in red and boldface, subgap peaks of cations in dark blue, and subgap peaks of anions in light blue.

ionization. This shift is similar for all species considered here and gets smaller as the chains get longer. Absorption energies of higher energy peaks differ for pyrroles and thiophenes, as they arise at least partly from 1 to $1^{\prime}$ transitions and are therefore related to the band gap of the neutral forms. Therefore, pyrrole cations have these peaks at higher energies than oligothiophene cations. As fluorination slightly increases the energy gaps of neutral thiophene oligomers and decreases those of neutral pyrrole oligomers, it shifts the $1-1^{\prime}$ related transitions in the cations and anions up for thiophene and down for pyrrole oligomers.

\section{Discussion}

Experimental data are available for $6 \mathrm{~T}$ and $6 \mathrm{DFT} .{ }^{14,15}$ The reduction potential increases by $0.45 \mathrm{eV}$; the oxidation potential, by $0.54 \mathrm{eV}$ upon fluorination. Calculated $\triangle \mathrm{SCF}$ EA and IP increase by 0.61 and $0.56 \mathrm{eV}$. Negative orbital energies give exactly the same result. Because IE and EA are lowered simultaneously, there is only a small electronic effect on the energy gap. The electrochemical band gap of 6DFT is $2.81 \mathrm{eV}$, the absorption maxima in dilute solution are $2.85 \mathrm{eV}$ for $6 \mathrm{~T}$ and $2.94 \mathrm{eV}$ for 6DFT. Thus the experimental band gap increases by $0.09 \mathrm{eV}$ upon fluorination. The TDB3P86-30\% excitation energies are $2.60 \mathrm{eV}$ for $6 \mathrm{~T}$ and $2.72 \mathrm{eV}$ for DFT, predicting an increase of $0.12 \mathrm{eV}$. Apart from a slight overestimation of the change in reduction potential, theory predicts changes upon fluorination very accurately.

Rotational barriers for thiophene and pyrrole dimers have been studied theoretically before. ${ }^{36,59-62}$ The results here are in good agreement with the literature. Fluorination changes the rotational profile, increasing the preference for trans forms and planarizing the transoid structures. In bipyrrole the large energetic effect is certainly due to the presence of $\mathrm{F}-\mathrm{H}$ bridges $\left(d_{\mathrm{F}-\mathrm{H}}=2.38 \AA\right)$ replacing steric repulsions between $\mathrm{H}$-atoms in unsubstituted pyrrole oligomers. In bithiophene, the reason for planarization is less obvious. Planarization of alkoxysubstituted thiophenes was attributed to $\mathrm{S}-\mathrm{O}$ interactions ${ }^{63,64}$ but a recent theoretical paper showed that such interactions are unlikely and that planarization is probably due to increased $\pi$-conjugation resulting from $\pi$-donations by oxygen lone pairs. ${ }^{65}$ A similar effect is expected upon fluoro-substitution since $\mathrm{F}$ is a $\pi$-donor as well. In addition, $F$ exerts strong inductive effects. 

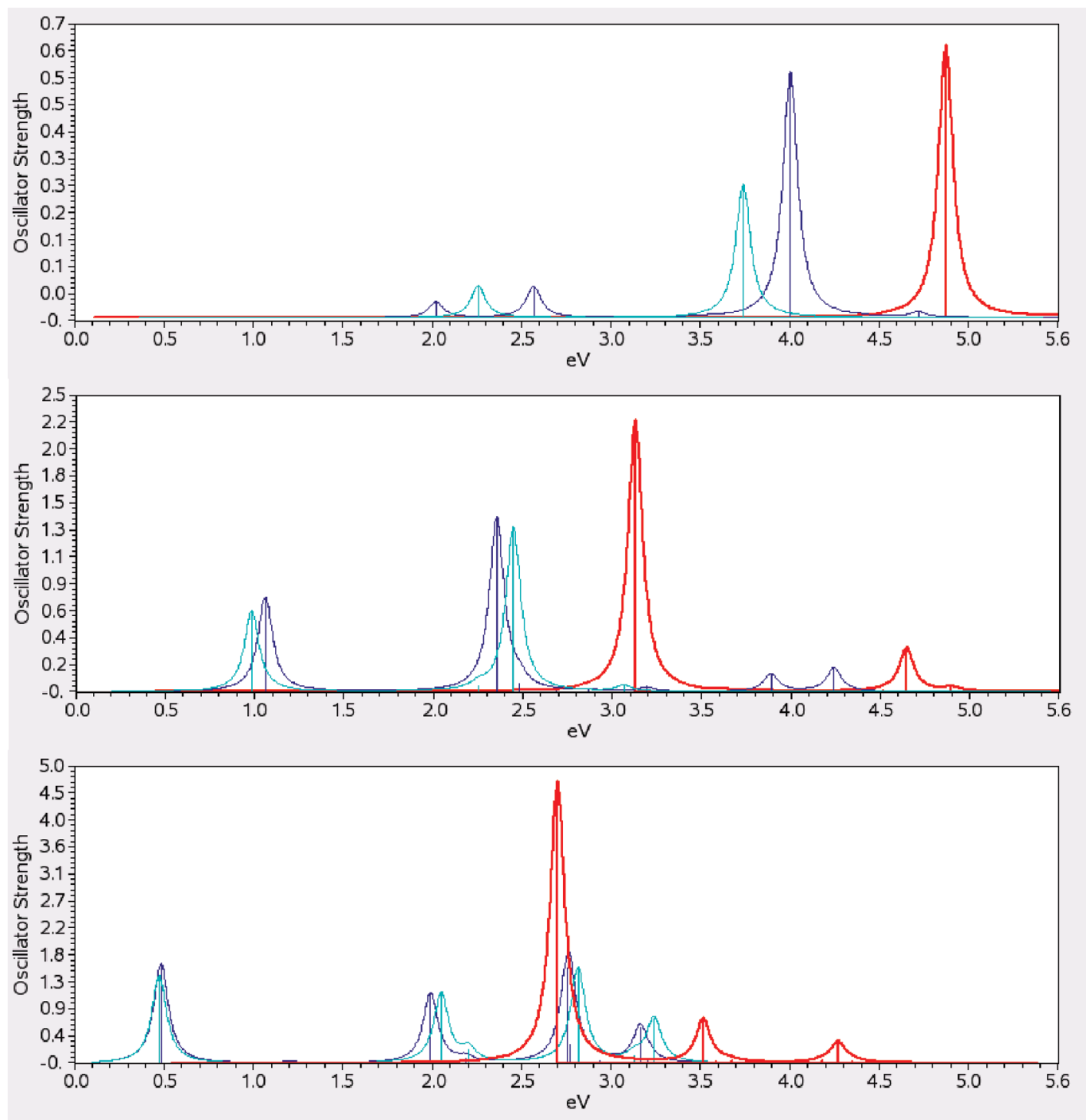

Figure 10. UV-absorption spectra of (top) $2 \mathrm{DFP}, 2 \mathrm{DFP}^{+}$, and $2 \mathrm{DFP}^{-}$, (middle) $6 \mathrm{DFP}, 6 \mathrm{DFP}^{+}$, and $6 \mathrm{DFP}^{-}$, and (bottom) $12 \mathrm{DFP}, 12 \mathrm{DFP}{ }^{+}$, and $12 \mathrm{DFP}^{-}$at the TDB3P86-30\%/6-31G* level of theory. All neutral species are shown in red and bold face, subgap peaks of cations in dark blue, and subgap peaks of anions in light blue.

Comparison of bond length in parent and fluorinated oligomers shows that all bonds are shortened upon fluorination. For thiophene oligomers the inter-ring and intra-ring single bonds decrease by 0.007 and $0.005 \AA$, the double bonds shorten by $0.005 \AA$. For pyrrole oligomers the single bonds are shortened the most, by 0.012 and $0.013 \AA$, the double bonds are shortened by only $0.001 \AA$. Bond length shortening was also observed experimentally for 6DFT. ${ }^{14,15}$ Thus conjugation is increased in all cases, especially between rings. Since the planarization energy of thiophene oligomers is very small, slightly increased conjugation can account for planarization.

Thiophene oligomers that are only slightly bent in the gas phase are planar in the solid state. ${ }^{66,67}$ Pyrrole oligomers are twisted at any chain length in the gas phase and PP is known to be more disordered than PT. ${ }^{68,69}$ In agreement with experiment ${ }^{14}$ the present results predict a slight band gap increase in PDFT compared to that for planar PT. This is, however, due to electronic effects and not due to steric repulsions between $F$ and $S,{ }^{14,15}$ as fluorinated oligomers are planar. Since pyrrole oligomers are planarized upon fluorination, disorder in the solid state is predicted to decrease. This explains why the band gap of PDFP is reduced by $0.6 \mathrm{eV} .{ }^{11}$ As shown in the Results, the theoretical band gap decrease of $0.58 \mathrm{eV}$ is partly due to planarization $(\sim 0.4 \mathrm{eV})$ and partly due to increased conjugation ( $0.2 \mathrm{eV})$.

The electronic effect of fluoro-substitution on band gaps is quite small because EA and IE are increased simultaneously. The major contribution to energy gap changes comes from geometric effects, which depend on the system and the positions of the fluorine atoms. Substitution across single bonds leads to twisting as fluorine atoms repel one another. Such twisting can lead to large increases in band gaps, as observed for polyphenylenevinylenes and thienylenevinylenes. ${ }^{17}$ For PDFT and PDFP only planarization is predicted and observed.

The lowering of energy levels upon fluorination is strongest for low lying levels and decreases for the high lying levels. As a result, the lowest $\pi$-band is contracted, the valence band is merely shifted down, and the conduction band is expanded. Thus perfluorinated polymers have low-lying wide conduction bands and are therefore predicted to allow high electron mobility along the polymer chain, as found experimentally for perfluorinated oligo(p-phenylenes)s $s^{12}$ and oligofluorenes. ${ }^{13}$ Recently, high electron mobility was also reported for dicyanomethylenesubstituted species. ${ }^{70}$ However, the best electron mobilities were 

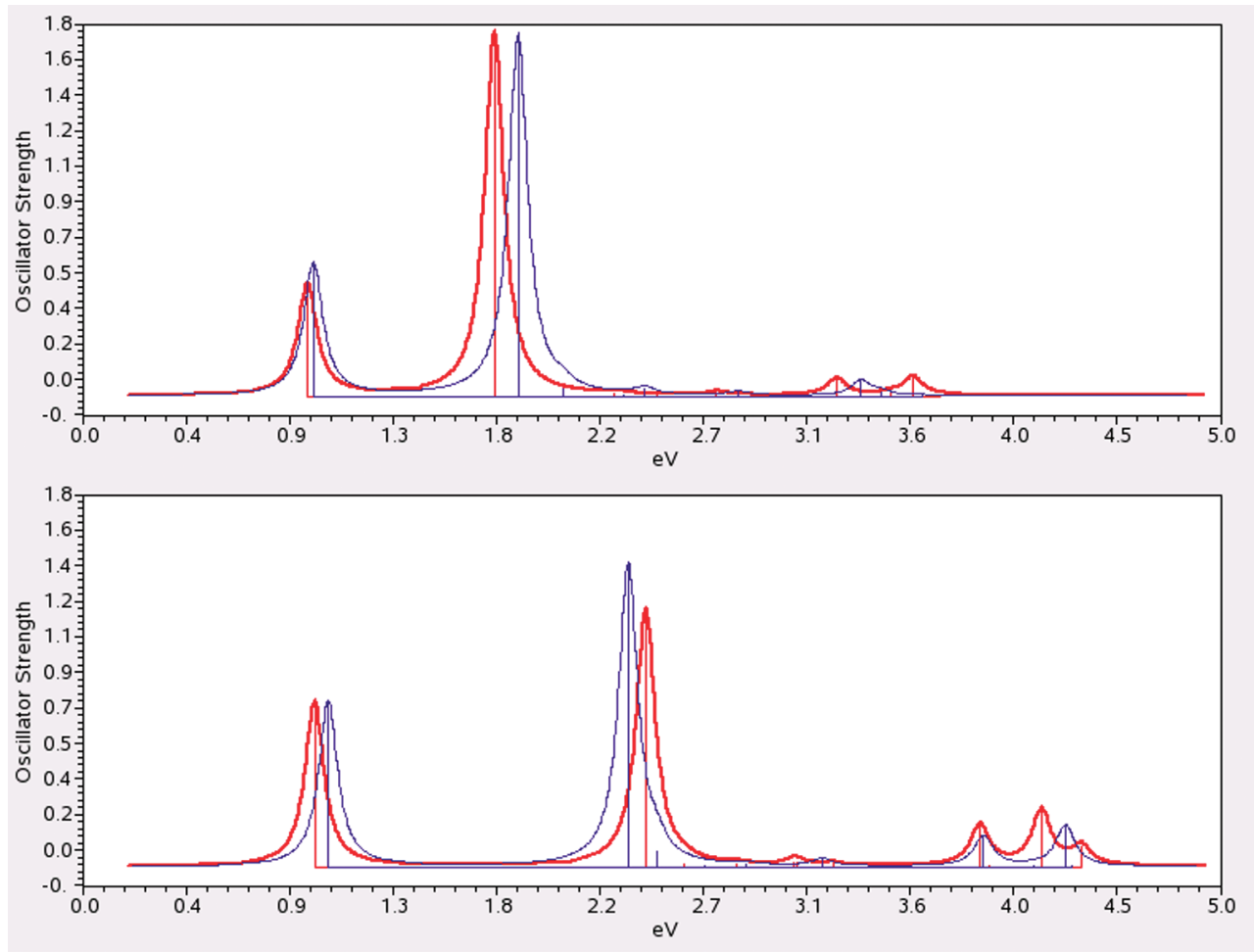

Figure 11. Comparison between $6 \mathrm{~T}^{+}$and $6 \mathrm{DFT}^{+}$(top) and $6 \mathrm{P}^{+}$and $6 \mathrm{DFP}^{+}$(bottom). Unsubstituted forms in red.

obtained for thin films of monomers, and mobilities decreased upon polymerization. This recalls the findings on a donoracceptor copolymer with a dicyanomethylene-substituted acceptor where electron mobility was found to be 500 times less than hole mobility. ${ }^{71}$ The difference between cyano- and fluorosubstitution arises because fluorination increases EAs by the electron withdrawing inductive effect of fluorine, while cyano groups increase EAs by providing a low lying empty $\pi$-system. ${ }^{9}$ Since this $\pi$-system tends to be localized on the CN group, conjugation along the polymer chain is low in the valence band, leading to low electron mobility along the polymer chain.

Another striking effect upon fluorination was the increased doping level that can be achieved in PDFP of 55\%. Audebert et al. ${ }^{11}$ reasoned that fluorine accepts some of the positive charge upon doping. Taking bithiophene cations as models for the $50 \%$ doping level, NBO analysis confirms that the decrease in electron density on the inner two fluorine atoms in 2DFP is $50 \%$ larger than that of inner two $\mathrm{H}$-atoms in $2 \mathrm{P}(0.042$ e vs $0.028 \mathrm{e})$.

The effect of fluorination on cation and anion spectra is rather small. There is no geometric effect as all charged species are planar. The electronic effect is also small since fluorination affects valence and conduction band edges to a similar degree. The fluorine $\pi$-orbitals lie much below the upper valence band edge and do not influence the spectra directly. Thus doping is predicted to have very similar effects on fluorinated conducting polymers as on their unsubstituted analogs.

\section{Conclusions}

The effect of fluorination on conducting polymers is best understood by separating geometric and electronic effects. The electronic effect upon fluorination is lowering valence and conduction band edges, thereby increasing IEs and EAs and increasing conduction band widths. Thus fluorinated polymers are harder to oxidize and easier to reduce. Combined with the increase of conduction band widths, fluoro-substitution is predicted to improve n-type conductivity by stabilizing n-doped forms and by improving electron mobility.

Since valence and conduction band edges are influenced similarly, electronic spectra of neutral and doped forms differ very little from those of the unsubstituted parents. Like unsubstituted oligomers, long fluorinated thiophene and pyrrole oligomers are predicted to have spectra different from those of short oligomers. At a chain length of about 12 rings, three almost equally strong peaks are expected, while short oligomers have only two sub-gap absorptions. As chain lengths increase, E2 tends to vanish and E3 dominates the spectra.

Geometric effects of fluorine substitution depend on structural details. If two fluorine atoms are separated by a single bond, steric repulsions will induce nonplanarity and the band gap increases. If fluorine is placed suitably close to $\mathrm{N}-\mathrm{H}$ units, hydrogen bridges induce planarity and the band gap decreases. The inductive effect of fluorine shortens all bonds and increases $\pi$-conjugation. In PDFT, the geometric effect is small and the band gap increases slightly. In PDFP hydrogen bridges cause planarization, improved order, and a band gap decrease of $0.6 \mathrm{eV}$.

Acknowledgment. This work was supported by Bilkent University, by TÜBITAK (TBAG-109T426), and by EU Seventh Framework Programme Project Unam-Regpot Grant No. 203953.

Supporting Information Available: Cartesian coordinates. Plots for extrapolating oligomer data to infinite chain length ( $\triangle \mathrm{SCF}$ IPs, negative HOMO energies, $\triangle \mathrm{SCF}$ EAs, negative LUMO energies). This material is available free of charge via the Internet at http://pubs.acs.org.

\section{References and Notes}

(1) Yamabe, T.; Tanaka, K.; Terama-E, H.; Fukui, K.; Shirakawa, H.; Ikeda, S. Synth. Met. 1980, 1, 321. 

43.

(2) Bakhshi, A. K.; Ladik, J.; Liegener, C.-M. Synth. Met. 1987, 20,

(3) Springborg, M. J. Am. Chem. Soc. 1999, 121, 11211.

(4) Karpfen, A. J. Phys. Chem. A 1999, 103, 2821.

(5) Salzner, U. J. Phys. Chem. B 2003, 107, 1129.

(6) Salzner, U. Synth. Met. 2003, 135-136, 311.

(7) Salzner, U.; Lagowski, J. B.; Poirier, R. A.; Pickup, P. G. J. Org. Chem. 1999, 64, 7419.

(8) Salzner, U.; Kiziltepe, T. J. Org. Chem. 1999, 64, 764.

(9) Salzner, U.; Köse, M. E. J. Phys. Chem. B 2002, 106, 9221.

(10) Gould, G. L.; Eswara, V.; Trifu, R. M.; Castner, D. G. J. Am. Chem. Soc. 1999, 121, 3781 .

(11) Audebert, P.; Miomandre, F.; Di Magno, S. G.; Smirnov, V. V.; Hapiot, P. Chem. Mater. 2000, 12, 2025.

(12) Heidenhain, S. B.; Sakamoto, Y.; Suzuki, T.; Miura, A.; Fujikawa,

H.; Mori, T.; Tokito, S.; Taga, Y. J. Am. Chem. Soc. 2000, 122, 10240.

(13) Okhubo, K.; Sakamoto, Y.; Suzuki, T.; Tsuzuki, T.; Kumaki, D.;

Tokito, S. Chem.-Eur. J. 2008, 14, 4472.

(14) Sakamoto, Y.; Komatsu, S.; Suzuki, T. J. Am. Chem. Soc. 2001, 123,4643

(15) Sakamoto, Y.; Komatsu, S.; Suzuki, T. Synth. Met. 2003, 133134,361 .

(16) Han, C. C.; Chen, H. Y. Macromolecules 2007, 40, 8969.

(17) Piacenza, M.; DellaSala, F.; Farinola, G. M.; Martinelli, C.; Gigli, G. J. Phys. Chem. B 2008, 112, 2996.

(18) Antonio, C.; Carmela, M.; Vita, P.; Francesco, B.; Maria, L.; Giovanni, B.; Pinalysa, C.; Francesco, N.; Gianluca, M. F. J. Polym. Sci.

Part A: Polym. Chem. , 48, 285.

(19) Middlecoff, J. S.; Collard, D. M. Synth. Met. 1997, 84, 221.

(20) Facchetti, A.; Deng, Y.; Wang, A.; Koide, Y.; Sirringhaus, H.;

Marks, T. J.; Friend, R. H. Angew. Chem., Int. Ed. Engl. 2000, 39, 4547.

(21) Krebs, F. C.; Jørgensen, M. Macromolecules 2002, 35, 7200.

(22) Facchetti, A.; Mushrush, M.; Yoon, M.-H.; Hutchison, G. R.;

Ratner, M. A.; Marks, T. J. J. Am. Chem. Soc. 2004, 126, 13859.

(23) Facchetti, A.; Yoon, M.-H.; Stern, C. L.; Hutchison, G. R.; Ratner,

M. A.; Marks, T. J. J. Am. Chem. Soc. 2004, 126, 13480.

(24) Letizia, J. A.; Facchetti, A.; Stern, C. L.; Ratner, M. A.; Marks,

T. J. J. Am. Chem. Soc. 2005, 127, 13476.

(25) Ie, Y.; Umemoto, Y.; Kaneda, T.; Aso, Y. Org. Lett. 2006, 8, 5381

(26) Yoon, M.-H.; Facchetti, A.; Stern, C. E.; Marks, T. J. J. Am. Chem Soc. 2006, 128, 5792.

(27) Ie, Y.; Nitani, M.; Ishikawa, M.; Nakayama, K.-I.; Tada, H.;

Kaneda, T.; Aso, Y. Org. Lett. 2007, 9, 2115.

(28) Umemoto, Y.; Ie, Y.; Saeki, A.; Seki, S.; Tagawa, S.; Aso, Y. Org.

Lett. 2008, 10, 1095 .

(29) Perdew, J. P. Phys. Rev. B 1986, 33, 8822.

(30) Salzner, U.; Pickup, P. G.; Poirier, R. A.; Lagowski, J. B. J. Phys.

Chem. A 1998, 102, 2572.

(31) Salzner, U. J. Chem. Theor. Comput. 2007, 3, 1143.

(32) Okur, S.; Salzner, U. J. Phys. Chem. A 2008, 112, 11842.

(33) Zade, S. S.; Bendikov, M. Org. Lett. 2006, 8, 5243.

(34) Alkan, F.; Salzner, U. J. Phys. Chem. A 2008, 112, 6053.

(35) Duarte, H. A.; Duani, H.; De Almeida, W. B. Chem. Phys. Lett. 2003, 369,114 .

(36) Millefiori, S.; Alparone, A. J. Chem. Soc., Faraday Trans. 1998 $94,25$.

(37) Salzner, U.; Baer, R. J. Chem. Phys., in press.

(38) Runge, R.; Gross, E. K. U. Phys. Rev. Lett. 1984, 52, 997.

(39) Bauernschmitt, R.; Ahlrichs, R. J. Chem. Phys. 1996, 104, 9047.

(40) Casida, M. E.; Jamorski, C.; Casida, K. C.; Salahub, D. R. J. Chem. Phys. 1998, 108, 4439.

(41) Stratmann, R. E.; Scuseria, G. E.; Frisch, M. J. J. Chem. Phys. 1998, 109, 8218 .
(42) Frisch, M. J.; Trucks, G. W.; Schlegel, H. B.; Scuseria, G. E.; Robb, M. A.; Cheeseman, J. R.; Montgomery, J. A., Jr.; Vreven, T.; Kudin, K. N.; Burant, J. C.; Millam, J. M.; Iyengar, S. S.; Tomasi, J.; Barone, V.; Mennucci, B.; Cossi, M.; Scalmani, G.; Rega, N.; Petersson, G. A.; Nakatsuji, H.; Hada, M.; Ehara, M.; Toyota, K.; Fukuda, R.; Hasegawa, J.; Ishida, M.; Nakajima, T.; Honda, Y.; Kitao, O.; Nakai, H.; Klene, M.; Li, X.; Knox, J. E.; Hratchian, H. P.; Cross, J. B.; Bakken, V.; Adamo, C.; Jaramillo, J.; Gomperts, R.; Stratmann, R. E.; Yazyev, O.; Austin, A. J.; Cammi, R.; Pomelli, C.; Ochterski, J. W.; Ayala, P. Y.; Morokuma, K.; Voth, G. A.; Salvador, P.; Dannenberg, J. J.; Zakrzewski, V. G.; Dapprich, S.; Daniels, A. D.; Strain, M. C.; Farkas, O.; Malick, D. K.; Rabuck, A. D.; Raghavachari, K.; Foresman, J. B.; Ortiz, J. V.; Cui, Q.; Baboul, A. G.; Clifford, S.; Cioslowski, J.; Stefanov, B. B.; Liu, G.; Liashenko, A.; Piskorz, P.; Komaromi, I.; Martin, R. L.; Fox, D. J.; Keith, T.; Al-Laham, M. A.; Peng, C. Y.; Nanayakkara, A.; Challacombe, M.; Gill, P. M. W.; Johnson, B.; Chen, W.; Wong, M. W.; Gonzales, C.; Pople, J. A. Gaussian 03; Gaussian Inc.: Wallingford, CT, 2004.

(43) Ipatov, A.; Cordova, F.; Doriol, L. J.; Casida, M. E. J. Mol. Struct.: THEOCHEM 2009, 914, 60.

(44) Cai, Z.-L.; Sendt, K.; Reimers, J. R. J. Chem. Phys. 2002, 117, 5543.

(45) Dreuw, A.; Weisman, J. L.; Head-Gordon, M. J. Chem. Phys. 2003, 119,2943

(46) Parac, M.; Grimme, S. Chem. Phys. 2003, 292, 11

(47) Salzner, U. Curr. Org. Chem. 2004, 8, 569.

(48) van Faassen, M.; de Boeji, P. L. J. Chem. Phys. 2004, 121, 10707.

(49) Dreuw, A.; Head-Gordon, M. Chem. Rev. 2005, 105, 4009.

(50) Hirata, S.; Head-Gordon, M. Chem. Phys. Lett. 1999, 302, 375.

(51) Hirata, S.; Lee, T. J.; Head-Gordon, M. J. Chem. Phys. 1999, 111, 8904

(52) Salzner, U. J. Chem. Theor. Comput. 2007, 3, 219

(53) Salzner, U. J. Phys. Chem. A 2008, 112, 5458.

(54) Luo, Y.; Ruud, K.; Norman, P.; Jonsson, D.; Ågren, H. J. Phys. Chem. B 1998, 102, 1710.

(55) Allouche, A.-R. Gabedit, http://gabedit.sourceforge.net, 2007.

(56) Pariser, R. J. Chem. Phys. 1956, 24, 250.

(57) van Haare, J. A. E. H.; Havinga, E. E.; van Dongen, J. L. J.; Janssen,

R. A. J.; Cornil, J.; Brédas, J. L. Chem.-Eur. J. 1998, 4, 1509.

(58) Campbell, D. K.; Bishop, A. R.; Fesser, K. Phys. Rev. B 1982, 26, 6862

(59) Alemán, C.; Julia, L. J. Phys. Chem. 1996, 100, 1524.

(60) Belletete, M.; Césare, N. D.; Leclerc, M.; Durocher, G. Chem. Phys. Lett. 1996, 250, 31.

(61) Duarte, H. A.; dos Santos, D. A.; Rocha, W. R.; de Almeida, W. B. J. Chem. Phys. 2000, 113, 4206.

(62) Raos, G.; Famulari, A.; Marcon, V. Chem. Phys. Lett. 2003, 379, 364.

(63) Raimundo, J.-M.; Blanchard, P.; Gallego-Planas, N.; Mercier, N.; Ledoux-Rak, I.; Hierle, R.; Roncali, J. J. Org. Chem. 2001, 67, 205.

(64) Roncali, J.; Blanchard, P.; Frere, P. J. Mater. Chem. 2005, 15, 1589.

(65) Poater, J.; Casanovas, J.; Sola, M.; Aleman, C. J. Phys. Chem. A

2009, 114, 1023

(66) Horowitz, G.; Bachet, B.; Yassar, A.; Lang, P.; Demanze, F.; Fave, J.-L.; Garnier, F. Chem. Mater. 1995, 7, 1337.

(67) Fichou, D.; Teulade-Fichou, M.-P.; Horowitz, G.; Demanze, F. Adv. Mater. 1997, 9, 75.

(68) Yakushi, K.; Lauchlan, L. J.; Clarke, T. C.; Street, G. B. J. Chem. Phys. 1983, 79, 4774

(69) Zhou, M.; Heinze, J. Electrochim. Acta 1999, 44, 1733.

(70) Usta, H.; Risko, C.; Wang, Z.; Huang, H.; Deliomeroglu, M. K.; Zhukhovitskiy, A.; Facchetti, A.; Marks, T. J. J. Am. Chem. Soc. 2009, 131,5586

(71) Huang, H.; Pickup, P. G. Chem. Mater. 1998, 10, 2212.

JP1005633 